\title{
Correction: Statistical analysis of continuous outcomes from parallel- arm randomized controlled trials in nutrition-a tutorial
}

\section{Christian Ritz (B)}

Published online: 14 October 2020

(c) The Author(s), under exclusive licence to Springer Nature Limited 2020

Correction to: European Journal of Clinical Nutrition https://doi.org/10.1038/s41430-020-00750-z
In the original version of this article, the legends to Figs. 2, 3 and 4 were inadvertently swapped. This has now been corrected in the PDF and HTML versions of the article. 